\title{
Castilla-La Mancha, paradigma de la crisis del periodismo: de los viejos magnates a los nuevos micromedios
}

\author{
Castilla-La Mancha, the journalism crisis sample: from press \\ barons to new diminished media
}

\author{
Dra. Belén Galletero Campos, Universidad de Castilla-La Mancha (España) \\ belen.galletero@uclm.es|http://orcid.org/0000-0002-9549-9507
}

\begin{abstract}
Resumen
En la última década se han producido cambios que afectan de lleno a los medios locales, aquejados por la caída de la publicidad, la irrupción de las redes sociales como canal distribuidor y la hegemonía en la difusión gratuita de los contenidos. Ante estos retos, los grandes grupos de comunicación han diversificado su actividad, desde la apuesta por reforzar el negocio audiovisual hasta la incursión en el comercio on line, pero ¿qué ocurre con aquellas iniciativas en manos de pequeños empresarios locales? Las encontramos en Castilla-La Mancha, territorio marcado por la ausencia de inversores fuertes que respalden la obligada transición hacia el entorno digital. En el sector de la prensa el resultado no deja lugar a dudas: de los dieciséis diarios que se editaban en 2006 sólo quedan tres. ¿Por qué la crisis ha afectado de una manera tan directa a estos medios? Algunas debilidades estructurales como su estrecha relación con el poder y su dependencia de las sistemáticas aportaciones de las instituciones han revelado su vulnerabilidad ante un momento adverso. Frente a ello, lo más característico son las nuevas iniciativas digitales impulsadas por periodistas despedidos de medios tradicionales. El censo elaborado por el Observatorio MediaCom de la Universidad de Castilla-La Mancha para 2017 muestra un número elevado de medios pero el análisis de su evolución reciente y su estructura, objeto de este trabajo y respaldado a partir de la revisión documental y entrevistas en profundidad, revela limitaciones que invitan a repensar fórmulas para el mantenimiento del periodismo de calidad.
\end{abstract}




\begin{abstract}
During last decade important changes have affected local media, upset by advertising fall, the creation of social media as a distribution channel and the hegemony in the free content spreading. According to these challenges, main communication groups have diversified their activity, from strengthening audiovisual business to incorporating online commerce, but what about those projects whose owners are small local enterprises? This is the situation of Castilla-La Mancha, a context distinguished by the lack of strong investment to support the necessary transition to the digital environment. Local newspapers evolution shows no doubt: form sixteen published in 2006 there are only three left. Why has the crisis affected these media in such a hard way? Some structural weaknesses such as their close relationships with governments and their dependence on the systematic contributions of public institutions have revealed their vulnerability in economic recession. At the same time, prevailing media are new digital initiatives promoted by journalists dismissed from traditional media. The census completed in 2017 by the Observatory of the University of Castilla-La Mancha MediaCom shows a high number of media but the analysis of their recent evolution and their structure, subject of this investigation and supported by documentary review and personal interviews, reveals limitations that invite to rethink strategies for the maintenance of quality journalism.
\end{abstract}

Palabras clave: Crisis, transformación, empresa periodística, Castilla-La Mancha, editor.

Keywords: Crisis, transformation, media enterprise, Castilla-La Mancha, editor.

\title{
1. INTRODUCCIÓN
}

Se cumplen diez años desde que la famosa caída de Lehman Brothers hiciera tambalearse a la economía mundial y diera al traste con la burbuja inmobiliaria española. Un periodo en el que el sector de los medios de comunicación, directamente afectado por los ciclos económicos (Sánchez-Tabernero y Pérez-Latre, 2012), no ha terminado de esclarecer cuál será la fórmula que le reportará garantías de futuro. Aún nos encontramos inmersos en una fase de transición en la que los viejos medios, en especial la prensa, que apura las últimas estrategias para preservar el soporte impreso, tratan de adaptarse a unos hábitos marcados por el consumo continuo, con un tiempo de exposición a los medios sin pauta fija pero en permanente estado de alerta (Díaz Nosty, 2013). Los medios nativos digitales, en cambio, sin el lastre de las grandes estructuras productivas, experimentan con fórmulas innovadoras de financiación (García Avilés y González 
Esteban, 2012) que ayuden a vencer la creciente aversión a pagar por contenidos en el entorno on line (Vara et al., 2017).

Castilla-La Mancha constituye un ejemplo claro de ese momento de impasse: los diarios en papel agonizan hasta tal punto que sólo quedan tres diarios provinciales y ninguno en Cuenca y Guadalajara; y por otro lado, los nuevos medios digitales se han erigido como la mejor fórmula de autoempleo para los periodistas. Sin embargo, se caracterizan por sus cortas trayectorias y sus exiguas plantillas, además de sus dificultades para progresar hacia su consolidación. Este trabajo se propone recoger la transformación empresarial experimentada en la estructura mediática de esta región en el último decenio. Nos hallamos ante una comunidad autónoma con baja tradición de pago por la información y preeminencia del consumo audiovisual, la que presenta el menor índice de difusión de toda España, con apenas 19 periódicos vendidos por cada mil habitantes - frente a los 42 del promedio nacional - de los cuales el 48\% corresponde a prensa nacional y el $32 \%$ a deportiva (AMI, 2018). El interés en indagar sobre estos cambios se sustenta en que el estudio de la estructura (Reig, 2009) contextualiza cualquier investigación posterior sobre las consecuencias que puede tener la migración acelerada que se está produciendo hacia el entorno digital (Laguna et al., 2016). Aunque las nuevas tecnologías reducen los costes de los productos culturales, las ideas y el raciocinio residen en las personas (Gallardo 2011), un activo cuyo precio permanece invariable con independencia del soporte. En este estudio partimos de la hipótesis de que en Castilla-La Mancha los problemas que han aquejado a los medios tradicionales - en especial al sector de la prensa y la televisión - no sólo no han desaparecido en el nuevo escenario digital, sino que se han acrecentado, a pesar de que el periodista tiene hoy más libertad de maniobra y más medios a su alcance para ser su propio editor (Casero-Ripollés, 2016).

La pregunta que subyace es, si el ciudadano no paga, ¿quién asumirá los costes de un periodismo profesional? ¿Qué ocurre cuando el periodismo no es una actividad rentable? Como se plantea el periodista Ignacio Ramonet (2011:14), “¿no le queda acaso más remedio para seguir existiendo que recurrir a subvenciones de mecenas, de fundaciones o del Estado?". Mientras el ciudadano tiene reconocido el derecho constitucional a recibir información veraz, en España desde que se inició la progresiva liberalización y desregularización del sector a partir de los 80 (Laguna y Martínez, 2013), las empresas periodísticas han caminado hacia el modelo americano del libre mercado, gobernadas por la ley de la oferta y la demanda. El estudio de caso que abordamos sugiere reconsiderar el papel de las administraciones en la promoción tanto de la creación de información de calidad como de su consumo. Planteamos la reflexión en la creencia de que los nutrientes mediáticos tienen un reflejo directo en la salud democrática y 
en la participación de la ciudadanía en la esfera pública (Díaz Nosty, 2005). Así, los gobiernos deben promover la alfabetización mediática definida como "la capacidad de acceder a los medios de comunicación" pero también la de "comprender y evaluar con criterio diversos aspectos de los mismos y de sus contenidos" (Recomendación 2009/625/CE). Puesto que el trabajo de los informadores se desarrolla en el seno social, se establece una correlación entre calidad de los medios de comunicación y calidad de la democracia (Rodríguez Borges, 2014). Analizar la crisis y la evolución de los primeros es paso previo a la reflexión acerca de sus efectos sobre la segunda.

\section{METODOLOGÍA}

Este trabajo parte de una primera aproximación exploratoria para completar el actual mapa de medios en Castilla-La Mancha que se desarrolló durante los años 2016 y 2017 en el seno del Observatorio de la Comunicación de la Universidad de Castilla-La Mancha, liderado por el investigador Antonio Laguna. Al cotejar los datos con aquellos localizados y referidos a una etapa previa a la crisis, podemos determinar que la evolución en los últimos años es la siguiente:

\begin{tabular}{|c|c|c|c|}
\hline CLM & 2008 & 2017 & Var. \\
\hline Diarios & 15 & 3 & $-80 \%$ \\
\hline Publicaciones no diarias & 46 & 36 & $-22 \%$ \\
\hline Radio & 64 & 117 & $83 \%$ \\
\hline Televisiones & 34 & 17 & $-50 \%$ \\
\hline Medios digitales & 10 & 63 & $530 \%$ \\
\hline TOTAL & 169 & 236 & $40 \%$ \\
\hline
\end{tabular}

El descenso más brusco se ha producido en el sector de los diarios, no tanto en las publicaciones no diarias que se localizan en su mayoría en el entorno rural y, a pesar de las cortas tiradas (en algunos casos se sitúan en los 500 ejemplares), todavía cumplen una importante función de referencia en el ámbito comarcal. Las emisoras de radio, repartidas entre lo público (el $44 \%$ de las 89 generalistas son municipales) y el paraguas de las grandes emisoras nacionales (34\%), han mostrado cierta estabilidad aunque bajo el aparente crecimiento se encuentra la implantación de emisoras de radiofórmula, sin que su puesta en marcha haya precisado la contratación de 
periodistas. Por otro lado, buena parte de las televisiones locales se ha visto afectada por el tránsito a la televisión digital terrestre en 2010, y de las cuatro que obtuvieron una licencia autonómica para emitir a través de TDT sólo queda una, ya que las otras tres pertenecían a los principales grupos periodísticos de Castilla-La Mancha que cerraron entre 2012 y 2013. El número más llamativo es el que representa a los digitales, con un crecimiento del $530 \%$ respecto a la reducida oferta de 2008, lo que confirma la tardía penetración de Internet en esta comunidad autónoma. En los 63 medios contabilizados no se han tenido en cuenta aquellos que tienen otro medio matriz (versiones digitales de periódicos, radios y televisiones) sino sólo aquellos que podrían considerarse hiperlocales por estar geográficamente definidos, orientados a la comunidad, originariamente nativos en la web y destinados a cubrir las lagunas percibidas en la cobertura de un área (Metzgar, Kurpius y Rowley, 2011).

Tomando como base el censo actualizado, en este trabajo se aborda un análisis descriptivo de la transformación empresarial experimentada en los medios en el contexto de recesión. Este objetivo requiere, por un lado, indagar en la evolución reciente de las empresas editoras de diarios ya que alrededor de la prensa surgieron los principales grupos locales de comunicación, al igual que en el ámbito nacional (Reig, 1998). Para ello, parte de la revisión de fuentes hemerográficas. Por otro lado, se ha aplicado al censo de digitales una ficha de análisis con datos empresariales como el año de inicio de actividad, el modelo de negocio, la fórmula empresarial y el tamaño de la plantilla. A partir de la observación directa, de los datos recogidos en el Registro Mercantil y la información que proporciona la auditoría de visitas OJD Interactiva, se ha conformado una radiografía de la empresa informativa que predomina hoy en esta región. Aplicando la triangulación metodológica, el estudio se ha complementado con entrevistas en profundidad a periodistas y promotores de medios. Esta técnica, aplicada a una muestra compuesta por diez editores y que se puede considerar representativa al apreciarse la saturación en el discurso (Serbia, 2007), completa el análisis cuantitativo y permite indagar en aquellos aspectos relativos al funcionamiento interno de las redacciones y a las rutinas profesionales (De Miguel, 2005). Su uso ha posibilitado extraer las limitaciones empresariales que asumen los nuevos editores.

\section{CRISIS DE LOS MEDIOS, ¿CRISIS DEL PERIODISMO?}

De la crisis coyuntural y estructural de los medios de comunicación han dado cuenta numerosos títulos editados en los últimos años, escritos tanto por profesionales (Bassets, 2013; Rius, 2016) como abordados desde la academia. Entre las causas de la crisis, Francisco Campos Freire (2010) identifica factores de tipo financiero como la restricción de crédito, la paralización de las inversiones y la recesión de la demanda; cambios tecnológicos derivados de remediación digital; y superación 
del modelo editorial basado en la actualidad. Sin embargo, comprender la situación actual precisa contemplar las transformaciones político-económicas que han conducido a la globalización neoliberal (De Mateo et al., 2010). La búsqueda del beneficio fácil y la sobreproducción tuvieron su apogeo en los primeros 2000 cuando las empresas de comunicación, los grandes grupos pero también los pequeños empresarios, tuvieron amplios márgenes de beneficios (García Santamaría, 2016).

Esta visión mercantilista de los medios no es algo reciente. Según Rosario De Mateo, Laura Bergés y Marta Sabater (2009:10) habría que remontarse al primer tercio del siglo XX para situar "la eclosión de la comunicación-negocio", con la influencia del entretenimiento en los contenidos y las inversiones de la empresa privada y la banca como apoyo económico, tanto a través de la publicidad como en la participación empresarial. En el contexto global, son mayoría los grupos de comunicación que tienen a la banca y a los grupos de inversión entre su cartera de accionistas (Labio, 2017). El periodista Pere Rusiñol encarna una de las voces más críticas hacia este modelo: "Este es patrón que ha operado en el capitalismo. Vale para casi todos los sectores empresariales que se movían en este marco de gigantismo alimentado por el crédito fácil. $Y$ entre ellos, por supuesto, los medios de comunicación (Rusiñol, 2013: 10). El público no ha sido ajeno a este fenómeno. Lo demuestra la depauperación de la imagen pública de los medios, otro de los efectos de la crisis. Tal como recoge el estudio Diez años que cambiaron los medios 2007-2017 coordinado por Bernardo Díaz Nosty (2017), España es uno de los tres países europeos, junto con Chipre y Grecia, en los que todos los medios de comunicación han perdido credibilidad. Lo llama "la anomalía española", ya que aunque los nuevos medios - en esta categoría se inscriben internet y las redes sociales - pueden parecer una alternativa ante el desprestigio continuado de los medios tradicionales, se da la paradoja de que su inclusión aumenta el déficit de confianza.

Algunos autores (Salaverría, 2012) sostienen que el periodismo es ahora más necesario que nunca para mostrar la senda entre la avalancha de datos que, de manera gratuita, están al alcance de la mano pero el estudio de los modelos comerciales en el ámbito local produce algunas incertidumbres. Los datos de Castilla-La Mancha apuntan hacia un medio digital low cost, encarnado en un periodista multitarea que intenta desarrollar algunos temas propios, al igual que en un diario al uso, pero los complementa con una oferta cuatro veces mayor de información de tipo corporativo e institucional que proviene directamente de los gabinetes. Todo ello con el agravante de que el medio digital ahora es ahora sustituto de la prensa local y su único sistema regulador es el mercado. 


\section{EL MODELO DE EMPRESA PERIODÍSTICA: EN LA PERMANENTE BÚSQUEDA DE LA RENTABILIDAD}

Dos síntomas avalarían las dificultades de rentabilizar la empresa periodística en esta región. El primero son las escasas inversiones que los grandes grupos españoles de comunicación han realizado en medios castellanomanchegos. No sucede así en el caso de las emisoras de radio, donde un tercio corresponde a las tres grandes cadenas (Onda Cero, SER y COPE), ya sea en propiedad directa o a través de empresas asociadas. Lo puede explicar la menor infraestructura necesaria para mantener una desconexión limitada de ámbito local, además de una política lógica de tener presencia en todas las capitales de provincia. En la prensa, en cambio, el proceso de concentración iniciado en los años 80 fue la salvación de aquellos medios que necesitaban sanear las cuentas y afrontar una renovación tecnológica (Gómez Calderón, 2006), circunstancias que las cabeceras castellanomanchegas tuvieron que afrontar en solitario.

El bajo interés por implantar o adquirir cabeceras que han mostrado los conglomerados mediáticos, incluso aquellos con importantes divisiones en prensa local como Vocento, Zeta y Prensa Ibérica, podría tener su raíz en la fuerte implantación de la prensa nacional (en la última década, los tres diarios más vendidos son de ámbito nacional) y en unos índices de ventas a la cola de todo el país, con cabeceras locales con una difusión media de 3.000 ejemplares. En los años 90 hubo algunos intentos de penetración en este mercado por parte de INPRESA, sociedad editoria del Grupo 16, como el cuadernillo provincial Nuevo Diario de Cuenca que se embuchaba en Diario 16 y que se mantuvo durante dos años, y la edición regional de Diario 16 desde 1994 hasta 1997.

La Verdad de Albacete, edición de la cabecera interregional con sede en Murcia, fue la excepción. Perteneció a Editorial Católica, posteriormente a Corporación de Medios de Comunicación S.A., germen del Grupo Correo, y finalmente a Vocento, al igual que la edición de $A B C$ para Castilla-La Mancha, que permanece hoy. Sin embargo, el holding de comunicación cerró el diario albaceteño en el año 2103 dentro de su Plan de Eficiencia, poniendo fin a cuarenta años de trayectoria.

Con esta excepción, el grueso del mercado periodístico en Castilla-La Mancha ha estado en manos de pequeños empresarios locales. Esto plantea un mercado muy distinto al que existe, por ejemplo, en Extremadura, una comunidad que también ha registrado un índice de difusión por debajo de la media española pero donde los dos diarios más vendidos son de identidad regional (AMI, 2018). Allí el grupo Vocento ha emprendido en los últimos años con la cabecera Hoy, líder en difusión, una estrategia de hiperlocalización para crear plataformas digitales, complementadas 
con publicaciones, dirigidas al medio rural (Marcos, 2017). En Castilla-La Mancha el soporte económico de un grupo fuerte habría facilitado sinergias dirigidas a ofrecer un producto más competitivo. El único grupo foráneo presente en la comunidad es el burgalés Promecal, que en el año 2002 adquiere las cinco Tribunas provinciales editadas por empresarios locales. En la actualidad ostenta el monopolio de la prensa diaria aunque sus tres periódicos se venden conjuntamente con La Razón, el diario más vendido.

El segundo de los síntomas de la difícil rentabilidad es la dependencia de los ingresos que proceden de la publicidad institucional. En Castilla-La Mancha no se han convocado ayudas directas a medios de comunicación y, por tanto, las aportaciones de las administraciones públicas se producen sólo a través de la publicidad institucional con el consiguiente cuestionamiento de sus sistemas de reparto.

Pongamos algunos ejemplos. En la siguiente tabla se recogen aquellas cantidades aportadas por organismos públicos en concepto de publicidad en los cuadernillos monográficos del Grupo El Día, que editaba cinco periódicos.

\section{Tabla 2. Magnitudes expresadas en euros de la publicidad inserta en monográficos Grupo El Día}

\begin{tabular}{|c|c|c|c|c|c|c|}
\hline Sector & 2004 & $\begin{array}{l}\% \text { DEL } \\
\text { TOTAL }\end{array}$ & 2008 & $\begin{array}{l}\% \text { DEL } \\
\text { TOTAL }\end{array}$ & 2010 & $\begin{array}{l}\% \text { DEL } \\
\text { TOTAL }\end{array}$ \\
\hline Organismos oficiales & 163.878 & $34,6 \%$ & $384.591,86$ & $50,6 \%$ & $142.375,22$ & $42,6 \%$ \\
\hline$-\mathrm{JCCM}$ & $51.951,15$ & $10,9 \%$ & $91.389,37$ & $12 \%$ & 24.747 & $7,4 \%$ \\
\hline TOTAL & $473.745,37$ & & 759.408 & & $334.308,33$ & \\
\hline
\end{tabular}

Fuente: Archivo privado administrativo del Grupo

El crecimiento que se produce entre los años 2004 y 2008 es intenso, alcanzándose el pico en el momento anterior a la crisis económica. Ese año estas aportaciones, en las que destaca la Junta de Comunidades como principal anunciante, superan la mitad de los ingresos publicitarios en los cuadernillos temáticos.

Un segundo caso ilustrativo es el de Promecal. La revista Noticias de la Comunicación recoge en su último número la cifra neta de negocio de dos empresas propiedad del grupo, La Tribuna de Toledo S.L. y La Tribuna de Ciudad Real S.A.. Si extraemos a partir del Portal de Transparencia de la Junta de Comunidades las cantidades invertidas en ambos diarios, obtenemos que la aportación de un solo anunciante, la administración regional, alcanza el 31,72\% de la cifra neta de negocio del diario de Toledo y un $26,8 \%$ en el caso del diario ciudadrealeño. Estamos, por tanto, ante un volumen de ingresos importante que, además, se complementa con aquel que procede 
de otras entidades como las diputaciones y ayuntamientos, aunque en este caso los importes no son públicos.

Por último, la siguiente tabla nos presenta una panorámica de los diez digitales que han recibido mayores inversiones publicitarias de la Junta en 2017. Lo primero que llama la atención es que el ranking en los importes no coincide con el que arrojan las visitas, siendo la audiencia, en principio, el principal criterio. Así, medios como EnCastilla-La Mancha.es recibe una cantidad superior a El Digital Castilla-La Mancha, teniendo tres veces menos visitantes; en cambio, CLM24 es el medio líder en audiencia (aunque no ofrece contenido de producción propia, sólo distribuye información de agencias) pero obtiene unos ingresos por debajo de los otros nueve medios. Si nos fijamos en el tamaño de la redacción, podría interpretarse que, excepto en el caso de Voces de Cuenca, la cantidad sufragada por la Junta podría cubrir casi al completo los gastos de personal.

Tabla 3. Elaboración propia a partir de los datos del Portal de Transparencia de la JCCM y OJD Interactiva

\begin{tabular}{|c|c|c|c|c|}
\hline Medios digitales & $\begin{array}{l}\text { Publicidad } \\
\text { JCCM } 2017\end{array}$ & $\begin{array}{l}\text { Audi } \\
\text { Visitas diar } \\
\text { medio }\end{array}$ & mpo & $\begin{array}{l}\text { Tamaño de } \\
\text { la redac- } \\
\text { ción }\end{array}$ \\
\hline ENCASTILLALAMANCHA.ES & 91.850 & 9.034 & $00: 45$ & 6 \\
\hline ELDIGITALCASTILLALAMANCHA.ES & 80.469 & 32.698 & 01:07 & 3 \\
\hline ELDIGITALDEALBACETE.COM & 87.160 & 46.990 & 01:21 & 6 \\
\hline ELDIARIO.ES - CLM & 67.897 & $\ldots$ & $\ldots$ & 4 \\
\hline LACERCA.COM & 67.457 & 25.132 & 01:33 & 2 \\
\hline PERIODICO CLM & 48.824 & $\cdots$ & $\cdots$ & $s / d$ \\
\hline ELDIADIGITAL.ES & 47.264 & 8.251 & $00: 48$ & 3 \\
\hline VOCES DE CUENCA & 28.902 & 18.333 & $06: 44$ & 7 \\
\hline DCLM.ES & 27.600 & 1.201 & $01: 25$ & 2 \\
\hline CLM24.ES & 22.732 & 102.336 & 01:12 & 7 \\
\hline
\end{tabular}

Lo expuesto viene a confirmar las serias dificultades económicas de los medios para subsistir, sabiendo que "donde empieza la publicidad acaba la libertad de expresión del periodista" (Reig, 2011:291), más aún si ésta procede de los gobernantes. 


\subsection{Los dueños del periodismo local}

Ante la ausencia de grandes inversores, los medios de comunicación han estado en manos de un reducido grupo de empresarios de la tierra. El 16 de marzo de 2009 El Mundo publicaba una información titulada "Los promotores acorralan a CCM" en la que daba cuenta del agujero generado por 20 empresarios "procedentes del ladrillo" que debían 3.316 millones a la caja de ahorros autonómica. Entre los principales deudores, tres empresarios de la comunicación: Domingo Díaz de Mera, Ignacio Barco y Antonio Miguel Méndez Pozo. A ellos se añade la sociedad CR Aeropuertos, promotora del polémico aeropuerto de Ciudad Real, que contaba entre sus accionistas a Santiago Mateo, otro de los principales nombres de la comunicación. En este caso, el cambio en la titularidad de los medios y el fenómeno de la financiarización (Rusiñol, 2013) estaba directamente relacionado con el sector inmobiliario y sus especulaciones. Para comprender la tipología de editor que ha predominado en este mercado abordaremos cuatro casos.

El más importante de estos empresarios es Santiago Mateo, un conquense propietario de discotecas que en 1984 funda el periódico El Día de Cuenca al quedarse la provincia sin diario cuando cerró Diario de Cuenca, perteneciente a la Cadena de Medios de Comunicación Social del Estado. A pesar de no contar con formación periodística, Mateo ejerció tanto la presidencia del Consejo de Administración como la dirección del diario, marcando las directrices editoriales. Lo que comenzó siendo un modesto diario de provincias y una empresa familiar, en veintidós años se había expandido por las cinco provincias con sendos periódicos, un semanario económico y una televisión regional, con la particularidad de que controlaba toda la cadena productiva de la prensa, desde la rotativa hasta la distribución. Según una edición especial de El Día, publicada con motivo de la inauguración de unas nuevas instalaciones en 2006, el grupo alcanzaba entonces una plantilla de más 170 personas. Entre el círculo de amigos del editor se encontraba José Bono, presidente de Castilla-La Mancha entre 1983 y 2004, tal y como reconoce el propio Mateo en una entrevista personal: "Recuerdo que en el año 90 yo iba mucho a Toledo y tenía mucha empatía con Pepe [Bono]. Para mí fue un hombre de centro izquierda, honesto, trabajador, levantó a esta región que no existíamos". Sin embargo, la apuesta editorial del grupo hacia el PSOE y la beligerancia con la que trataron al Partido Popular en las elecciones autonómicas de 2011 terminó por ahogar las cuentas del grupo al alcanzar María Dolores de Cospedal la presidencia. El Grupo El Día declaró concurso de acreedores en el año 2013, tras varios meses de impagos a sus trabajadores. La nueva presidenta había recortado las aportaciones publicitarias que recibía el grupo para repartirlas de otro modo. 
En 2014 un reportaje de investigación del periodista Carlos Iserte para El Plural sacó a la luz el reparto publicitario del Gobierno regional del PP a través de la Fundación para la Promoción de Castilla-La Mancha, una entidad que, según él, contaba con sólo dos empleados y un presupuesto anual de entre 8 y 10 millones de euros cuyos únicos destinatarios eran los medios de comunicación.

Ilustración 1. Tabla publicada en el medio digital EI Plural.com el 15/ 12/2014

\begin{tabular}{|l|r|}
\hline EL TOP TEN PUBLICITARIO DE COSPEDAL EN CLM & EUROS \\
\hline EMPRESAS MÉNDEZ POZO (Promecal, La Tribuna, TV CyL, TV Navarra...) & 4.976 .785 \\
\hline TV POPULAR + GUADANEWS.ES & 2.588 .237 \\
\hline ABC & 1.968 .819 \\
\hline CADENA COPE & 1.736 .719 \\
\hline ONDA CERO & 1.002 .198 \\
\hline TV LA REGIONAL + TELETOLEDO & 748.870 \\
\hline CADENA SER & 301.050 \\
\hline EL DIGITAL DE CASTILLA-LA MANCHA & 198.770 \\
\hline PERIODISTA DIGITAL & 182.548 \\
\hline ECOS + ENCASTILLALAMANCHA.ES & 174.790 \\
\hline
\end{tabular}

Fuente: Fundación para la promoción de Castilla-La Mancha

Según estos datos, el mayor beneficiario durante la legislatura del PP fue Promecal, que en 2013, el momento más crítico para las empresas periodísticas, recuperó el proyecto de La Tribuna de Cuenca que había cerrado en 2009. En su inauguración, la presidenta calificaba su reaparición como una "gran noticia para la libertad, el periodismo y para la provincia de Cuenca" (CastillaLa Mancha.es, 2013). De Méndez Pozo destacan sus buenas relaciones con gobernantes de distinto color. Cuando en 2006 inauguró en Burgos la nueva sede de su grupo acudió Juan Vicente Herrera, Presidente de la Comunidad de Castilla y León, del Partido Popular, pero también José María Barreda, presidente de Castilla-La Mancha, del Partido Socialista, y Juan Pedro Hernández Moltó, entonces presidente de la Caja Castilla- La Mancha. Recogemos un fragmento del reportaje de Juan Luis Sánchez en eldiario.es sobre las presiones que Méndez Pozo ejerció a través de sus medios a Manuel Pérez Castell, alcalde de Albacete desde 1999 hasta 2008: 
Hacia el final de la segunda legislatura de Castell, cuando llegaba la precampaña electoral para las municipales de 2007, Miguel Méndez Pozo instó al alcalde a que le ayudase con uno de sus negocios con el ladrillo. "Me dijo que le tenía que recalificar una serie de terrenos que había comprado para hacerlos urbanizables", afirma hoy Pérez Castell. "Le dije que no". (Sánchez, 2014).

Según señala el digital, un testigo relató así el momento: "Durante la charla, Méndez Pozo recordó - como si hiciera falta - que era propietario de La Tribuna de Albacete e "insinuó al alcalde que no le convenía tener en contra al periódico durante la campaña", la de las municipales de 2007".

En Ciudad Real destacan los nombres de Domingo Díaz de Mera e Ignacio Barco. Este último estuvo implicado en la creación de La Tribuna de Ciudad Real en 1990. Domingo Díaz de Mera, por su parte, fue promotor de dos diarios de ámbito regional: Las Noticias de Castilla-La Mancha (1998-2000) y Metro Diario C-LM (2005-2009), ambos de corta permanencia y enmarcados en periodos electorales. Díaz de Mera, abogado de profesión, comenzó sus negocios en la comunicación con varias emisoras de la Cadena Ser en la provincia de Ciudad Real, donde también editó durante algún tiempo la revista Crónicas de la Mancha. Los medios del empresario se agruparon bajo la sociedad Green Publicidad y Medios S.A., que en el año 2002 entra en el accionariado del Grupo El Día, asociándose a Santiago Mateo para impulsar El Día de Ciudad Real. Además, el holding, que ya había obtenido un buen número de licencias comarcales de TDT en Andalucía (Reig, 2013), consiguió una de las autonómicas para Castilla-La Mancha para su televisión regional Canal Regional de Noticias. Díaz de Mera fue compañero de colegio del socialista José María Barreda, presidente de Castilla-La Mancha entre 2004 y 2011. Además, fue presidente del Club Balonmano Ciudad Real, llevando al equipo a sus mayores éxitos, hasta 2011, momento en que lo traslada a Madrid para convertirlo en el Atlético alegando falta de apoyo en su tierra. Ocurre unos meses después de las elecciones y casi al tiempo que el cierre de su televisión regional y de El Día de Ciudad Real. El periodista Carlos Otto, ex redactor del diario ciudadrealeño, explicaba en El Confidencial el funcionamiento editorial del medio:

Con la entrada en la dirección de Cristina Vives, sobrina del propio Díaz de Mera, la línea se radicalizó para defender a ultranza a Barreda y elogiar proyectos del propio Díaz de Mera, como el ahora ruinoso aeropuerto de Ciudad Real. Son muchos los redactores que recuerdan épocas informativas muy duras: No se podía sacar a Cospedal. A lo mejor podíamos decir 'la líder del PP en Castilla-La Mancha', pero no podíamos poner su 
nombre ni sacarla en ninguna foto. $\mathrm{Y}$ con el Aeropuerto y con Barreda igual: había que defenderlos sí o sí (Otto, 2011).

Otto fue despedido de El Día de Ciudad Real tras publicar en su blog personal un texto crítico con Díaz de Mera y con Juan Antonio León Triviño, presidente del Aeropuerto.

Por último, mencionaremos al empresario albaceteño Jose Manuel Martínez, presidente de Corporación HMS, un conglomerado de logística y materiales de automoción que incorpora en los años 2000 la televisión local Visión 6 y el periódico provincial El Pueblo de Albacete. Entrevistas mantenidas con algunos periodistas que trabajaron en ambos medios ponen de manifiesto que la peculiar línea editorial del diario, basaba en una serie de filias y fobias del editor que se reflejaban en el veto a determinadas personalidades del ámbito público albaceteño y el trato favorable a sus allegados. El cumplimiento de esta serie de imperativos llegó a derivar en presiones a los periodistas, circunstancia que impidió consolidar una plantilla estable. Las bajas voluntarias y despidos fueron habituales en los nueve años de vida del periódico. En 2015 la Asociación de Periodistas de Albacete decidió actuar de oficio y enviar varios contenidos emitidos por Visión 6 a la Comisión de Arbitraje, Quejas y Deontología del Periodismo de la FAPE, al considerar que el material audiovisual "sobrepasaba los límites aceptables en la profesión periodística".

El promotor de medios en Castilla-La Mancha destaca por su carácter local y sus vínculos con el poder. La ausencia de un sistema para regular la publicidad institucional ha propiciado la consolidación de medios aparentemente privados pero sujetos a las decisiones de las administraciones públicas y susceptibles a los cambios de gobierno.

\subsection{El nuevo ecosistema digital y la acelerada transición a la red}

El estudio de los entramados de poder es más complejo en los nuevos medios digitales, condicionado, entre otros factores, por la inestabilidad del entorno (Salaverría, 2007) y la variedad de fórmulas informativas (Avilés, 2017). Las limitaciones parten de la falta de transparencia, la ausencia de censos oficiales y el inexistente rastro que dejan los digitales ya extinguidos. En Castilla-La Mancha los primeros medios digitales fueron las versiones on line de ABC CLM y La Verdad de Albacete, ambas impulsadas por Vocento en 1998, y el primer medio nativo digital, La Crónica de Guadalajara (1999). Sin embargo, el desarrollo del ciberperiodismo en la comunidad fue lento, localizándose todavía un número muy reducido de digitales diez años después (Morate, 2010). Cabe pensar que el entorno on line no generó interés hasta que los periodistas tuvieron que asumir fórmulas alternativas de autoempleo. De los 63 digitales en 2017, el 44,4\% comenzó su actividad entre 2013 y 2015, momento que coincide con el cierre de los grandes grupos de 
comunicación (El Día, Green Publicidad y Medios) y empresas periodísticas de mayor tamaño (La Verdad de Albacete). El impulso que adquieren los digitales hiperlocales a partir de 2010 se explicaría por la generación de nuevos nichos a partir de vacíos en las coberturas geográficas y en los contenidos de los medios tradicionales; nuevos servicios on line como Wordpress o Youtube; y la explosión en la oferta de dispositivos digitales como móviles o tabletas, entre otras razones (Radcliffe, 2012).

\section{Tabla 4. Elaboración propia a partir de la información aportada por los medios}

\section{Evolución del número de medios digitales en Castilla-La Mancha por año de creación}

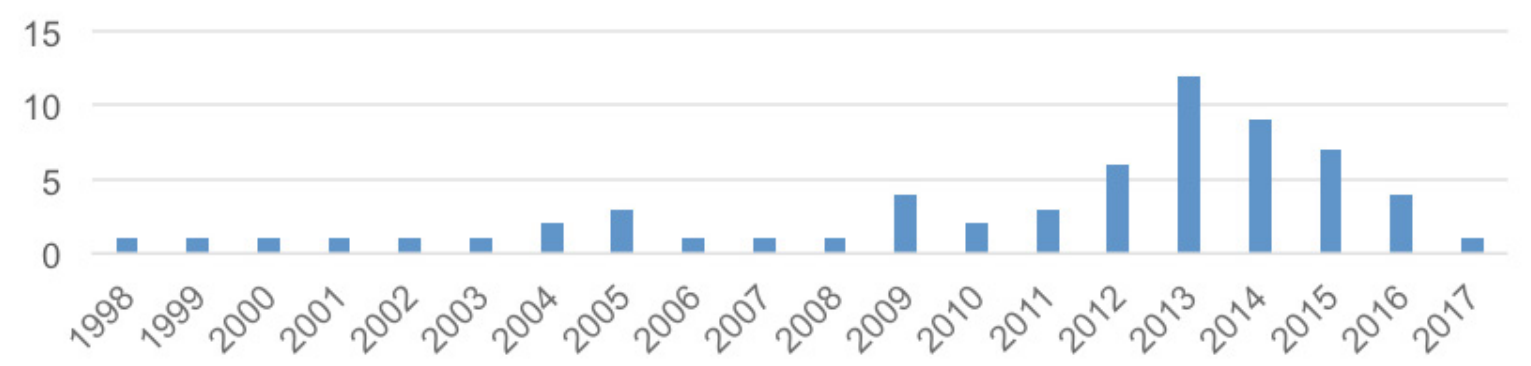

La información localizable a partir de la observación directa revela que el 17,5\% no aporta datos sobre su empresa editora y, por tanto, no se puede conocer su fórmula empresarial ni el tamaño de su plantilla. Tampoco a través de fuentes complementarias como OJD Interactiva puesto que no todos han solicitado su sistema de auditoría. Esto significa que en once digitales no se puede saber quién controla y gestiona la información, una opacidad que suscita preocupación. En los que es posible identificar la empresa editora, la fórmula predominante es la sociedad limitada, quedando en segundo lugar los autónomos. Las cooperativas o las comunidades de bienes son clara excepción, al igual que las alianzas. Sólo se identifica una fórmula de colaboración entre la edición autonómica del eldiario.es CLM y Las Noticias de Cuenca y Albacete Capital, que consiste en un intercambio no remunerado de información entre el medio regional y los locales.

Por otro lado, de los treinta y cuatro en los que se puede identificar la plantilla un $70 \%$ tiene entre 0 y 2 empleados y sólo cuatro superan la cifra de 6 . Nos encontramos, por tanto, ante una estructura atomizada de micromedios, compuestos por una o dos personas, que realizan información dirigida a su entorno local. 


\section{Tabla 5. Elaboración propia a partir de OJD Interactiva, Registro Mercantil y observación de los medios}

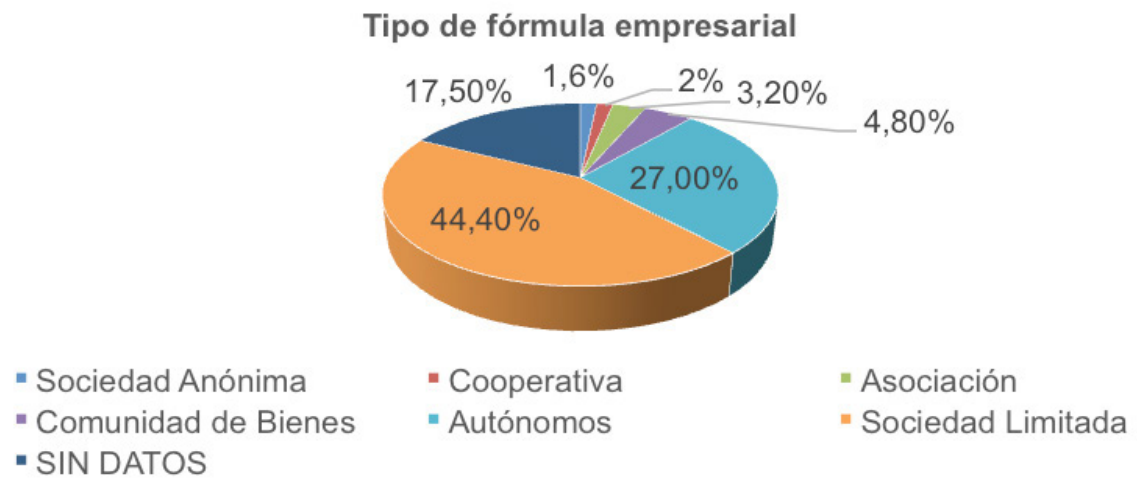

Su distribución territorial ratifica que son aquellas provincias con localidades de mayor tamaño y con mayor tejido industrial en sus comarcas - Toledo y Ciudad Real - las que agrupan mayor número de digitales. Al igual que sucede con los medios convencionales, Guadalajara y Cuenca destacan por sus carencias, con apenas 7 y 5 digitales, respectivamente.

\section{Tabla 6. Elaboración propia a partir del Registro Mercantil y observación de los medios}

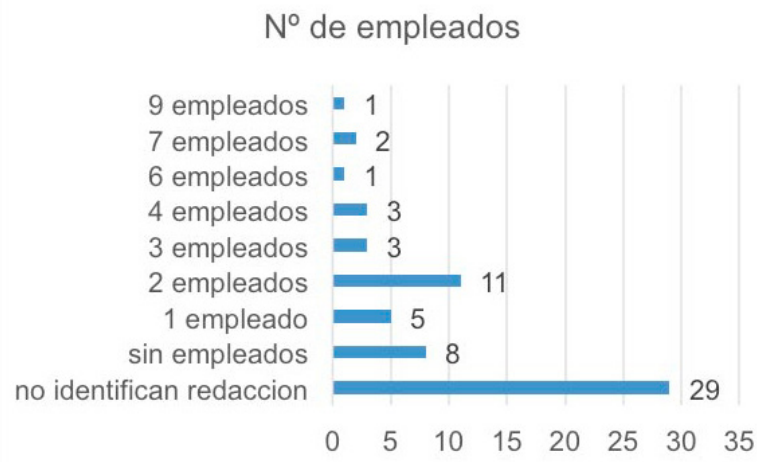

\subsection{El discurso del periodista emprendedor}

Aunque en la entrevista la información se recoge mediante enunciados verbales, susceptibles de interpretación subjetiva (Taylor y Bogdan, 1992), la reiteración de planteamientos que se aprecia puede ser indicativa de tendencias contrastadas. A partir de la transcripción de las entrevistas, se han sistematizado los resultados a través de una matriz de análisis que contemplaba las siguientes categorías: motivaciones para impulsar un medio digital; funcionamiento empresarial; 
vías de financiación y modelo de negocio; aspectos positivos y negativos del emprendimiento; valoración de la oferta informativa local y regional de Castilla-La Mancha; y perspectivas de futuro.

Destaca la unanimidad a la hora de expresar los motivos de emprendimiento: la crisis y las escasas perspectivas laborales. En los perfiles analizados, las acreditadas experiencias en otros medios conviven con las carencias en la formación empresarial y, por tanto, surgen dificultades en la gestión económica y comercial de las propuestas. Los emprendedores coinciden en las limitaciones que plantea el modelo de negocio cuya única fuente de ingresos es la publicidad. Aluden a la reticencia del anunciante local - el pequeño empresario o autónomo - sobre la eficacia de la publicidad digital, una desconfianza a la que no se enfrentarían los medios tradicionales.

La radiografía que aporta el análisis cuantitativo se confirma: "Son mini-medios, portales dirigidos por una persona. $Y$ si haces un seguimiento, prácticamente viven de la publicidad institucional, salvo alguna excepción". Y los relatos evidencian la debilidad de un mercado que se considera "saturado" o "sobreexplotado". La dependencia de la publicidad institucional no sólo no ha disminuido respecto a la de los periódicos, sino que ha aumentado, al no contar los digitales con la doble vía de financiación. "En época de vacas gordas, era una pata importante. Cuando llegaron las vacas flacas a algunos se les quedó en la única pata", indica un periodista. La crisis económica se tradujo en una pérdida de 14.700 empresas entre 2008 y 2015 en Castilla-La Mancha según el INE. Dos de ellos señalan intentos de promover un sistema de suscripciones de pago, con escaso éxito. Recordamos que se trata de un contexto en el que apenas existe hábito de pago por la información: predomina el consumo de televisión - el 54,7\% de la población la utiliza para informarse frente al 15,8\% que elige el periódico (CIS, 2015) - y el índice de rotación en prensa es el más alto de España (AEDE, 2012).

Se producen cambios en las rutinas, siendo el más significativo el teletrabajo, sobre el que los entrevistados apuntan aspectos positivos como la versatilidad, y otros negativos, como la inexistencia de un espacio de trabajo delimitado y compartido con otros compañeros. Con la desaparición de la redacción se pierde ese espacio de generación de debate, el marco propicio para desarrollar un periodismo crítico (Rius, 2016). Bajo los enunciados tópicos sobre el romanticismo de la profesión, se detectan jornadas laborales en las que no existe una delimitación clara entre lo personal y lo profesional, y dificultades para atender a la comunidad en red y mantener coberturas in situ. En otros casos, las condiciones de precariedad se manifiestan de manera explícita: "Pese a que económicamente estaba en su mejor momento, cerramos porque no era suficiente. Seguramente hemos hecho lo más difícil pero el coste personal ha pasado factura física y psíquicamente". 
Son varios los que defienden que el periodismo de calidad deberá pasar por el pago por la información, aunque se muestran pesimistas sobre las vías para implantarlo. Parece claro que para garantizar la continuidad de los proyectos será necesaria la experimentación en otros modelos de negocio que permitan superar el voluntarismo que revelan los promotores.

\section{DISCUSIÓN Y CONCLUSIONES}

A lo largo de este trabajo se han recorrido los cambios acontecidos en la empresa periodística en Castilla-La Mancha en los últimos diez años, siendo el aspecto más relevante la desaparición de los grandes grupos locales de comunicación, que empleaban a más de un centenar de personas, en pro de pequeños micromedios que fructifican en el entorno digital.

La principal limitación para investigar el funcionamiento económico de estos medios es la falta de transparencia y la imposibilidad de conocer las cuentas de todas estas pequeñas iniciativas para valorar cuál su situación financiera real. El panorama actual presenta un número elevado de digitales pero de gran inestabilidad y de corta trayectoria, salvo excepciones. Otro aspecto relevante es la vuelta a una situación de monopolio en el periodismo impreso, que no se había producido desde tiempos de la Transición. Así, en tres provincias la única voz periodística es la de La Tribuna, perteneciente a un grupo editorial en manos de un editor, Antonio Miguel Méndez Pozo, con unas particularidades que ya describimos. Por otro lado, en Guadalajara las tres publicaciones que se mantienen el bisemanario Nueva Alcarria y dos mensuales gratuitos siguen en manos del sector inmobiliario, en concreto, del Grupo Quabit, antes Rayet. Cuenca destaca por su escasa oferta periodística, constituyendo una zona informativamente deprimida.

La preocupación por la creciente precariedad, su influencia en la calidad periodística y la desintegración de las redacciones ha sido objeto de análisis por parte de la comunidad investigadora (Figueras et al, 2012; López Hidalgo et al., 2013; Soengas et al., 2014). Así, el escenario digital no puede ser sólo contemplado desde el determinismo que interpreta que la tecnología per se ha creado más y mejores medios. En lo que respecta a las condiciones laborales de los profesionales, la libertad que ha supuesto prescindir del mandato, a veces arbitrario, del editor se ve cercenada por las carencias en recursos materiales y humanos para desarrollar un medio de comunicación consolidado.

El consumo informativo de la sociedad castellanomanchega y la evolución que han experimentado sus medios obligan a repensar la función del periodista, desarrollando competencias de ayuda al desarrollo, no sólo aquellas que tienen que ver con lo mercantil o lo tecnológico. En ello deberían tener un papel decisivo las administraciones públicas, especialmente aquellas de ámbito local y regional. Queda probado que la publicidad institucional termina convirtiéndose en un 
método de subvención encubierta que, en el peor de los casos, se aplica de forma arbitraria pero, en el mejor, se distribuye en función de las visitas, por lo que tampoco discrimina la información exclusiva, propia y trabajada, sino que se mueve, de nuevo, por los criterios mercantiles de contabilizar el clic.

\section{REFERENCIAS}

Asociación de Medios de Información (2018). Libro Blanco de la Información 2017. Asociación de Editores de Diarios Españoles (2012). Libro Blanco de la Prensa 2011.

Avilés, R. (2017). La concentración del poder en el gran mundo digital. En Reig, R. y Labio, A. (Eds.). El laberinto mundial de la información, pp. 227-246. Barcelona. Antropos Editorial. Bassets, L. (2013). El último que apague la luz. Madrid: Taurus.

Campos Freire, F. (2010). Las empresas de medios de comunicación revisan y amplían sus modelos de negocio. Razón y Palabra, 15(74). http://www.razonypalabra.org.mx/N/N74/ VARIA74/16FreireV74.pdf

Casero Ripollés, A. (2016). El periodismo emprendedor ante el reto de su consolidación. Anuario ThinkEPI, v. 10, 203-208. https://recyt.fecyt.es//index.php/ThinkEPI/article/view/ thinkepi.2016.42

Castilla-La Mancha.es (2013). Cospedal califica la reaparición de La Tribuna de Cuenca como una "gran noticia para la libertad, el periodismo y para la provincia de Cuenca". Nota de prensa, 17/09/2013. Recuperado de: http://www.castillalamancha.es/actualidad/ notasdeprensa/cospedal-califica-la-reaparici\%C3\%B3n-de-la-tribuna-de-cuenca-comouna-\%E2\%80\%9Cgran-noticia-para-la-libertad-el-0

Centro de Investigaciones Sociológicas (2015). Estudio $n^{\circ} 3093$, Postelectoral Elecciones Autonómicas 2015. Castilla-La Mancha.

Comisión Europea (2012). Recomendación 2009/625/CE de 20 de agosto de 2009, sobre la alfabetización mediática en el entorno digital para una industria audiovisual y de contenidos más competitiva y una sociedad del conocimiento incluyente, DOUE, 29/8/2009, pp. 9-12. http://eur-lex.europa.eu/LexUriServ/LexUriServ.do?uri=OJ:L:2009:227:0009:0012:ES:PDF

De Mateo, R.; Bergés, L. y Sabater, M. (2009). Gestión de empresas de comunicación. Sevilla: Comunicación Social Ediciones y Publicaciones.

De Mateo, R; Bergés, L. y Garnatxe, A. (2010). Crisis, ¿qué crisis? Los medios de comunicación: empresas y periodismo en tiempos de crisis. En Campos Freire, F. (Coord.) El cambio mediático, pp.75-106. Zamora: Comunicación Social Ediciones y Publicaciones. 
De Miguel, R. (2005). La entrevista en profundidad a los emisores y los receptores de los medios. En Berganza, M.R. y Ruíz, J. (Coords.). Investigar en comunicación: Guía práctica de métodos y técnicas de investigación social en Comunicación, pp. 251-266. Madrid: McGraw Hill.

Díaz Nosty, B. (2005). El déficit mediático. Donde España no converge con Europa. Barcelona: Bosch.

Díaz Nosty, B. (2013). La prensa en el nuevo ecosistema informativo. ¡Que paren las rotativas! Madrid: Fundación Telefónica.

Díaz Nosty, B. (Coord.) (2017). Diez años que cambiaron los medios. 2007-2017. Madrid/Barcelona: Fundación telefónica/Ariel.

Figueras, M.; Mauri, M.; Alsius, S. y Salgado, F. (2012). La precariedad te hace dócil. Problemas que afectan a la profesión periodística. El profesional de la información, 21(1), 70-75.http:// dx.doi.org/10.3145/epi.2012.ene.09

Gallardo, F. (2011). Nuevos modelos de negocio para la prensa. TELOS. Cuadernos de Comunicación e Innovación. Madrid: Fundación Telefónica, 86, 76-86.

García Avilés, J. y González Esteban, J. L. (2012). Cibermedios nativos españoles: explorando modelos de rentabilidad. Trípodos, 30, 153-167.

García Santamaría, J. V. (2016). Los orígenes de la crisis de la prensa en España y las causas de su tardía transición digital. En Mateos, C. y Herrero, F.J. (Coords.) La pantalla insomne, pp. 841-855, Tenerife: Cuadernos Artesanos de Comunicación. http://www.revistalatinacs. org/15SLCS/2016 libro/039 Garcia.pdf

Gómez Calderón, B. (2006). Bonanza económica frente a estancamiento de la difusión. En Díaz Nosty, B. y Fernández Beaumont, J. (Coords.), Tendencias 06: Medios de comunicación. El año de la televisión, pp. 69-76. Madrid: Fundación Telefónica.

Labio, A. (2017). Contexto: la estructura mundial de la información. En Reig, R. y Labio, A. (Eds.), El laberinto mundial de la información, pp. 35-56. Barcelona. Antropos Editorial.

Laguna, A. y Martínez, F.A. (2013). De la dictadura al mercado: la Transición de los medios de comunicación en España o dónde quedó la responsabilidad social de los medios. Trípodos, 32, 171-184.

Laguna, A. et al. (2016). Los medios de comunicación en Castilla-La Mancha. Informe 2015 Mediacom. Cuenca: Servicio de Publicaciones Universidad de Castilla-La Mancha. https:// ruidera.uclm.es/xmlui/handle/10578/10285 
López Hidalgo, A.; Ufarte, M. J. y López Redondo, I. (Coords.) Del deterioro del periodismo tradicional al horizonte de las nuevas tecnologías. Sevilla: Cuadernos de Periodismo y Comunicación. Universidad de Sevilla.

Marcos, C. (2017) La televisión de proximidad en Extremadura. Sostenibilidad e identidad. Tesis doctoral. Universidad de Extremadura.

Metzgar, E.T.; Kurpius, D.D. y Rowley, K.M. (2011). Defining hyperlocal media: Proposing a framework for discussion. New Media \& Society, 13(5), 772-787. http://journals.sagepub. com/doi/pdf/10.1177/1461444810385095

Morate, I. (2010). Medios y cibermedios desde la perspectiva autonómica. Situación actual y tendencias en Castilla-La Mancha. Ciudad Real: Diputación de Ciudad Real.

Otto, C. (2011). Díaz de Mera cerrará su periódico 'El Día de Ciudad Real' tras las autonómicas. El Confidencial, 12/04/2011. Recuperado de: https://www.elconfidencial.com/ comunicacion/2011-04-12/diaz-de-mera-cerrara-su-periodico-el-dia-de-ciudad-real-traslas-autonomicas_513337/

Radcliff, D. (2012). Here and Now: UK Hyperlocal Media Today, NESTA. https://ssrn.com/ abstract $=3041668$

Ramonet, I. (2011). La explosión del periodismo. De los medios de masas a la masa de medios. Madrid: Clave Intelectual.

Reig, R. (1998). Medios de Comunicación y poder en España. Barcelona: Paidós Papeles de Comunicación.

Reig, R. (2009). Bases teóricas y documentales para el estudio de la Estructura de la Información y el análisis estructural de los mensajes. Estudios sobre el Mensaje Periodístico, 15, 385-407.

Reig, R. (2011). Los dueños del periodismo. Claves de la estructura mediática mundial y de España. Barcelona: Gedisa.

Reig, R. (2013). Educación para el mercado. Barcelona: Gedisa.

Rius, J. C. (2016). Periodismo en reconstrucción. De la crisis de la prensa al reto de un oficio más independiente y libre. Barcelona: Edicions de la Universitat de Barcelona.

Rodríguez Borges, R.F. (2014). ¿Crisis del periodismo y crisis de la democracia? Una reconsideración del oficio periodístico en el ecosistema digital. Dilemata, 14, 1-17. http://www.dilemata.net/ revista/index.php/dilemata/article/view/261

Rusiñol, P. (2013). Papel mojado. La crisis de la prensa y el fracaso de los periódicos en España. Barcelona: Debate. 
Salaverría, R. (2007). La investigación sobre ciberperiodismo en España: Tendencias, resultados y perspectivas. En Actas La convergencia digital en los medios de comunicación valencianos, Valencia, pp. 15-34. http://dadun.unav.edu/handle/10171/5096?locale=en

Salaverría, R. (2012). Medios y periodistas, ¿un futuro compartido? Cuadernos Evoca 7. El futuro del periodismo, pp. 11-14. http://dadun.unav.edu/handle/10171/22428

Sánchez, J. L. (2014). Méndez Pozo también presionó al alcalde de Albacete: 'Me pidió recalificaciones'. eldiario.es, 18/01/2014. Recuperado de: http://www.eldiario.es/political Exalcalde-Albacete-Mendez-Pozo-recalificara_0_219078611.html

Sánchez-Tabernero, A. y Pérez-Latre, F. (2012). Innovación en los medios: la ruta del cambio. Pamplona: EUNSA.

Serbia, J. M. (2007). Diseño, muestreo y análisis en la investigación cualitativa, Hologramática, 7, 123-146.

Soengas, X., Rodríguez Vázquez, A. y Abuín, N. (2014). La situación profesional de los periodistas españoles: las repercusiones de la crisis en los medios. Revista Latina de Comunicación Social, 69, 104-124.

Taylor, S. J. y Bogdan, R. (1992). Introducción a los métodos cualitativos en investigación. La búsqueda de los significados. Barcelona: Paidós.

Vara, A., Negredo, S. y Amoedo, A. (2017). Digital News Report España 2017. Navarra: Ediciones Universidad de Navarra. 